\title{
Tarifliche Weiterbildungspolitik in den Niederlanden und in Deutschland
}

Mit der gestiegenen Bedeutung der beruflichen Weiterbildung wurde Mitarbeiterqualifizierung auch zum Thema von Tarifverhandlungen. Bei der zentralen Frage der Weiterbildungsfinanzierung einigen sich die Tarifparteien in der Regel darauf, dass der Arbeitgeber die Kosten der betrieblichen Weiterbildung trägt. Nur in wenigen kleinen Branchen wird darüber hinaus auch die Errichtung eines überbetrieblichen Finanzierungsfonds geregelt, sodass es abgesehen von diesen Branchen bei einer einzelbetrieblichen Finanzierung bleibt. Ein Blick über die Landesgrenzen zeigt, dass eine tarifliche Fondsfinanzierung in den Niederlanden zum festen Bestandteil des Weiterbildungssystems gehört und eine über den Einzelbetrieb hinaus am Branchenbedarf orientierte Weiterbildungspolitik fördert.

KLAUS BERGER, DICK MORAAL

\section{Einleitung}

Während im internationalen Vergleich die Niederlande bei zentralen Indikatoren zur betrieblichen Weiterbildung auf den vorderen Plätzen rangieren, befindet sich Deutschland auf der internationalen Rangliste eher abgeschlagen auf den hinteren Rängen. Dieser Befund wird im vorliegenden Beitrag zum Anlass genommen, die institutionalisierte Kooperation von Tarifparteien und Staat in der beruflichen Weiterbildung näher zu betrachten. Ein zentrales Element stellen hierbei die tariflichen Branchenfonds zur Weiterbildungsfinanzierung dar. Während diese in der tariflichen Weiterbildungspolitik Deutschlands nur eine marginale Rolle spielen, bilden Branchenfonds in den Niederlanden das Fundament für eine branchenbezogene Koordination beruflicher Weiterbildungspolitik.

\section{Betriebliche Weiterbildung in \\ Deutschland und in den \\ Niederlanden}

Die betriebliche Weiterbildung ist der wichtigste Bereich der beruflichen Weiterbildung und eine der Hauptmaßnahmen zur fortwährenden Anpassung der Kompetenzen der
Beschäftigten an die Anforderungen der Wirtschaft. Im Vergleich mit Ländern, die eine ähnliche sozio-ökonomische Struktur aufweisen - wie die nord- und westeuropäischen Länder - ist die betriebliche Weiterbildung in Deutschland deutlich schlechter aufgestellt. (Behringer et al. 2008). Beim Vergleich der Performanz der betrieblichen Weiterbildung in den Niederlanden und Deutschland zeigt sich Folgendes:

(1) Das Weiterbildungsangebot der Unternehmen in Deutschland ist vergleichsweise gering. Die Unternehmen konzentrieren ihre betriebliche Weiterbildung auf relativ wenige Beschäftigte, die Dauer der Maßnahmen ist kurz und die direkten Aufwendungen je Teilnehmenden sind niedrig. Beim Angebot an arbeitsplatzintegrierten und arbeitsplatznahen Formen platziert sich Deutschland allerdings wesentlich besser. Die Professionalisierung der Weiterbildung in Unternehmen - zum Beispiel im Hinblick auf die Bedarfsermittlung, die Weiterbildungsplanung, die Aufstellung eines Weiterbildungsbudgets und die Evaluierung - lässt in Deutschland nur einen geringen Grad an Systematisierung erkennen.

(2) Die Unternehmen in den Niederlanden weisen bei den wichtigsten Indikatoren höhere Werte als die deutschen Unternehmen auf: Sie bieten überdurchschnittlich häufig betriebliche Weiterbildung in der Form von internen und externen Lehrveranstaltungen an. An diesem Angebot neh- 
men zwar relativ wenige Beschäftigte teil, die Intensität der betrieblichen Weiterbildungsmaßnahmen und die direkten Aufwendungen je Teilnehmenden sind allerdings hoch. Bei den arbeitsplatzintegrierten und arbeitsplatznahen Formen liegen die Unternehmen in Deutschland jedoch vor den niederländischen. Die Professionalisierung der Weiterbildung in niederländischen Unternehmen ist wie in Deutschland auch nicht sehr ausgeprägt.

In diesem Beitrag wird argumentiert, dass ein wichtiger Grund für die insgesamt gesehen bessere Performanz der betrieblichen Weiterbildung in den Niederlanden im Vergleich zu Deutschland auch in der überbetrieblichen Kooperation und Koordination von Staat und Tarifparteien in der beruflichen Weiterbildung liegt.

\subsection{Kooperation und Koordination in der beruflichen Weiterbildung}

Mit dem Ansatz der „Comparative Political Economy of Skill Formation (CPE-SF)“ entwickelte sich in den letzten Jahren eine Forschungsrichtung, die sich primär mit der Investition in und Entwicklung von (gesellschaftlichem) Humankapital und deren Zusammenhängen mit der Entwicklung anderer politisch-ökonomischer Institutionen wie dem Wohlfahrtsstaat, der Unternehmenskontrolle, den industriellen Beziehungen und Finanzsystemen beschäftigt. Das berufliche Bildungssystem in Deutschland als auch in den Niederlanden wird als ein System typisiert, in dem kooperativ und koordinierend die nötigen Qualifikationen und Fähigkeiten hergestellt werden (collective skill formation system). Die Akteure in der beruflichen Bildung (Staat und Tarifparteien) kooperieren in der Organisation, Durchführung, Finanzierung und Evaluierung (Busemeyer 2009; Busemeyer/Trampusch 2011; Trampusch/Eichenberger 2011). Zwischen den beiden Ländern existieren deutliche Unterschiede bei der Kooperation und Koordination der Tarifparteien und des Staates in der beruflichen Bildung. Während in Deutschland eine ausgeprägte, gesetzlich festgelegte Zusammenarbeit der Gewerkschaften und Arbeitgeberverbände in der beruflichen Ausbildung besteht, kooperieren diese Akteure bei der beruflichen Weiterbildung nur im Bereich der wenigen staatlich anerkannten Fortbildungsabschlüsse miteinander. In den Niederlanden arbeiten Staat und Tarifparteien auf der Grundlage allgemeingültig erklärter tariflicher Vereinbarungen sowohl in der beruflichen Ausbildung als auch in der Weiterbildung zusammen.

Grundsätzlich gilt für beide Länder, dass drei Teilbereiche der beruflichen Weiterbildung identifiziert werden können:

- der Bereich der betrieblichen Weiterbildung,

- der Bereich der individuellen beruflichen Weiterbildung, - der Bereich der Weiterbildung für Arbeitslose und von Arbeitslosigkeit bedrohte Arbeitnehmerinnen und Arbeitnehmer als Teil der aktiven Arbeitsmarktpolitik (Sauter 1989).
Länder lassen sich danach einteilen, ob das System der beruflichen Weiterbildung eher kooperativ ausgerichtet ist die einzelnen oben genannten Bereiche der beruflichen Weiterbildung sind institutionell miteinander verzahnt oder eher segmentiert - es existiert eine institutionelle Trennung zwischen den einzelnen Bereichen der beruflichen Weiterbildung.

In unterschiedlicher Weise sind z. B. in Dänemark und den Niederlanden die Akteure im Bereich der beruflichen Weiterbildung - Staat, Unternehmen, Gewerkschaften, Individuen und Bildungsträger - in ein komplexes System kombinierter Verantwortlichkeiten für die berufliche Weiterbildung eingebunden. Dagegen liegen z. B. in Deutschland und auch Österreich die Verantwortlichkeiten der beruflichen Weiterbildung bei den jeweiligen Akteuren selbst: In diesen Ländern ist der Staat verantwortlich für die Weiterbildung der Arbeitslosen und von Arbeitslosigkeit bedrohten Arbeitnehmerinnen und Arbeitnehmer, die Unternehmen für die betriebliche Weiterbildung und die Individuen für ihre eigene berufliche Weiterbildung. Das Prinzip der Subsidiarität (Verantwortung im eigenen Bereich) ist vor allem in Deutschland sehr stark entwickelt (Moraal 2007).

In einer vergleichenden Länderanalyse haben Trampusch/Eichenberger (2011) erstmalig systematisch den Einfluss von institutionellen und politischen Rahmenbedingungen auf die tarifliche Weiterbildungspolitik und ihre Finanzierung untersucht. Die Autoren stellen heraus, dass bei der Genese und Implementierung tariflicher Weiterbildungspolitik fünf Rahmenbedingungen eine wichtige Rolle spielen (Trampusch/Eichenberger 2011, S. 18):

- „das Vorhandensein einer unterstützenden und fördernden Rolle des Staates,

- die größere Bereitschaft kleiner Unternehmen und ihrer Organisationen, sektorale Qualifizierungstarifverträge abzuschließen,

- die Bereitschaft der Gewerkschaften, eher tarifliche Qualifizierungstarifverträge abzuschließen statt rechtliche Regelungen zu präferieren,

- die gemeinsame Bereitschaft der Tarifparteien, die Qualifizierung in den Lohnverhandlungen aufzunehmen sowie - die Konsens- und Kooperationskultur zwischen den Tarifparteien."

Diese fünf Rahmenbedingungen zeigen, dass es die Bereitschaft der Akteure (vor allem Staat und Tarifparteien) zur Kooperation und Koordination ist, die die Entstehung

\footnotetext{
Vgl. hierzu das BIBB-Forschungsprojekt „Berufliche Weiterbildung: Ursachen möglicher Unterinvestitionen und Anreize für Betriebe und Beschäftigte" - Arbeitspaket IV "Tarifliche Weiterbildungspolitik in Deutschland und in den Niederlanden“, in dem u .a. die Wirksamkeit umlagefinanzierter Tariffonds in Deutschland und den Niederlanden untersucht werden (https://www2.bibb.de/tools/fodb/pdf/ zw_23301.pdf; letzter Zugriff: 15.05.2012).
} 
ÜBERSICHT 1

\section{Entwicklung der Qualifizierungstarifverträge in Deutschland}

\begin{tabular}{ll} 
Die letzten 10 Jahre & $\begin{array}{l}\text { Eigenständige Qualifizierungstarifverträge, in denen z. B. Wei- } \\
\text { terbildungszeiten, Weiterbildungspflichten des Arbeitgebers, } \\
\text { Kostenübernahme oder Bedarfsermittlung geregelt wurden. }\end{array}$ \\
\hline 1980er/1990er Jahre & $\begin{array}{l}\text { Qualifikationsförderliche Gestaltung der Arbeitsorganisation } \\
\text { und Entgeltsysteme. }\end{array}$ \\
\hline 1970 er/1980er Jahre & $\begin{array}{l}\text { "Qualifizieren statt entlassen" - Verknüpfung betrieblicher Inves- } \\
\text { titionsplanungen mit vorausschauender Qualifizierungsplanung }\end{array}$ \\
\hline 1960 er/1970er Jahre & $\begin{array}{l}\text { Fortbildung und Umschulung zur Abfederung rationalisierungs- } \\
\text { bedingter Entlassungen }\end{array}$ \\
\hline
\end{tabular}

Quelle: BIBB-Forschungsprojekt „Berufliche Weiterbildung: Ursachen möglicher Unterinvestitionen

und Anreize für Betriebe und Beschäftigte“ - Arbeitspaket IV "Tarifliche Weiterbildungspolitik in

Deutschland und in den Niederlanden“, https://www2.bibb.de/tools/fodb/pdf/zw_23301.pdf

(letzter Zugriff 15.05.2012)

ÜBERSICHT 2

\section{Regelungsinhalte von Qualifizierungstarifverträgen in Deutschland}

\begin{tabular}{|c|c|}
\hline selten & $\begin{array}{l}\text { überbetriebliche Unterstützungsstrukturen } \\
\text { - Branchenfonds } \\
\text { - gemeinsame Institutionen zur Weiterbildungsförderung }\end{array}$ \\
\hline $\begin{array}{l}\text { selten und mit } \\
\text { Einschränkungen }\end{array}$ & $\begin{array}{l}\text { quantifizierte Weiterbildungsansprüche der Beschäftigten bei } \\
\text { Lohnfortzahlung }\end{array}$ \\
\hline häufig & $\begin{array}{l}\text { generelle Verfahrensweisen } \\
\text { - Verfahren zur Ermittlung des Weiterbildungsbedarfs } \\
\text { - Förderung spezifischer Beschäftigtengruppen } \\
\text { - Mitarbeitergespräche } \\
\text { - Zeiten für Weiterbildungsmaßnahmen } \\
\text { - Voraussetzung für den Abschluss von Betriebsvereinbarungen }\end{array}$ \\
\hline
\end{tabular}

Quelle: BIBB-Forschungsprojekt „Berufliche Weiterbildung: Ursachen möglicher Unterinvestitionen und Anreize für Betriebe und Beschäftigte" - Arbeitspaket IV "Tarifliche Weiterbildungspolitik in Deutschland und in den Niederlanden“, https://www2.bibb.de/tools/fodb/pdf/zw_23301.pdf (letzter Zugriff 15.05.2012). der Chemieindustrie eigenständige Qualifizierungstarifverträge abgeschlossen worden (vgl. z.B. Bahnmüller 2012). Der Regelungsschwerpunkt liegt beim Bedarfsermittlungs-, Planungs- und Gestaltungsprozess der betrieblichen Weiterbildung unter Beteiligung des Betriebsrats (Übersicht 2). Ein zentrales Instrument ist dabei der Anspruch der Beschäftigten auf ein regelmäßiges Qualifizierungsgespräch.

Bei der Mehrzahl der Qualifizierungstarifverträge kamen die Tarifparteien überein, dass betriebliche Weiterbildung einzelbetrieblich vom Arbeitgeber zu finanzieren ist, während bei „persönlicher beruflicher" Weiterbildung die Beschäftigten die Kosten zu tragen haben. Tarifliche Regelungen zur Einrichtung umlagefinanzierter Weiterbildungsfonds stellen eher die Ausnahme dar. So umfassen die Qualifizierungstarifverträge der letzten Jahre etwa 5 Mio. Beschäftigte (Bahnmüller/Hoppe 2011), in den Geltungsbereich umlagefinanzierter Tariffonds fallen jedoch nur knapp 100.000 Beschäftigte. Diese wurden in den kleineren Branchen des Gerüstbaugewerbes, der Textil- und Bekleidungsindustrie und bestimmten Tarifregionen der Landund Forstwirtschaft eingerichtet.

Bahnmüller (2009, S. 13) beschreibt drei Vorteile dieser überbetrieblichen Tariffonds. Durch die paritätische Beteiligung von Arbeitgebern und Gewerkschaften orientieren sich Finanzierungsentscheidungen weniger an Einzelinteressen, sondern mehr am Bedarf der gesamten Branche. Sie ermöglichen eine Weiterbildungsfinanzierung unabhängig von konjunkturellen Schwankungen und der aktuellen wirtschaftlichen Lage eines Betriebes. Gerade kleinere Einzelbetriebe werden über die Tariffondsverwaltungen nicht nur finanziell, sondern auch bei der Organisation von Weiterbildung entlastet.

Der Anspruch, Qualifizierung von Fachkräften nicht mehr als einzelbetriebliches Problem, sondern als Herausforderung für die gesamte Branche anzusehen, wird von den bestehenden Tariffonds unterschiedlich eingelöst. So wurde bei der Umsetzung des Qualifizierungsvertrags in der Textilund Bekleidungsindustrie von Arbeitgeberseite darauf verzichtet, „die Mittel auf Projekte und Maßnahmen zu lenken, die darauf ausgelegt sind, den künftigen Qualifikationsbedarf der Branche zu identifizieren und entsprechende Maßnahmen zu konzipieren und zu realisieren“" (Bahnmüller/ Jentgens 2005, S. 92). Hingegen verfolgt die Gewerkschaftsseite durchaus das Ziel, den branchenspezifischen Qualifikationsbedarf zu klären. Inwieweit der tarifvertragliche Finanzierungsfonds hierzu beitragen kann, scheint jedoch noch nicht festzustehen (Bahnmüller/Jentgens 2005, S. 93).

Die Orientierung am branchenspezifischen Qualifikationsbedarf war ein zentraler Regelungsgegenstand des Tarifvertrags über die Berufsbildung im Gerüstbaugewerbe. „So vereinbarten die Tarifparteien in dem Tarifabkommen nicht nur die Finanzierungsmodalitäten, sondern auch die Fortbildungsmaßnahmen (wie z. B. geprüfter Gerüstbau-Kolonnenführer), die über den Tariffonds gefördert werden sollten “ (Berger et al. 2012, S. 52). Einen branchenspezifischen Aspekt hat auch der Tariffonds in der Forstwirtschaft Nieder-
Tarifvereinbarungen, die in Deutschland Fragen der Quali fizierung regeln, reichen bis in die 1960er Jahre zurück (Übersicht 1). Im letzten Jahrzehnt sind insbesondere in den großen Tarifbereichen der Metall- und Elektroindustrie und

\section{Tarifliche Weiterbildungspolitik in Deutschland}


sachsens bzw. in der Land- und Forstwirtschaft SchleswigHolsteins. Hierüber werden nicht nur die Beschäftigten, sondern auch die Weiterbildung der Arbeitnehmer wird gefördert, die in der Branche beschäftigt waren und eine forstwirtschaftliche Tätigkeit fortsetzen wollen (Berger et al. 2012, S. 51; vgl. ausführlicher Übersicht 3 und Tabelle 1)

Der Vorteil der Konjunkturunabhängigkeit der Weiterbildungsfinanzierung besteht bei den drei Tariffonds insofern, als wirtschaftliche Krisen nicht unmittelbar auf die Weiterbildungsfinanzierung der Betriebe durchschlagen. Dennoch sind die zur Verfügung stehenden Fondsmittel auch von der Wirtschaftslage abhängig, weil die Beitragszahlungen an die betriebliche Beschäftigtenzahl bzw. an die Bruttogehälter geknüpft sind. Der Vorteil der überbetrieblichen Unterstützungsstrukturen für kleinere Betriebe wird am Beispiel des Gerüstbaugewerbes deutlich. Hier bietet die Tariffondsverwaltung (Sozialkasse des Gerüstbaugewerbes) neben der Weiterbildungsfinanzierung auch eine organisierte Kooperation mit überbetrieblichen Berufsbildungseinrichtungen an, die die Betriebe in die Lage versetzt, ihren Beschäftigten die erforderliche Fortbildung anzubieten (Berger et al. 2012, S. 52).

In den drei Branchen, in denen Tariffonds eingerichtet sind, werden sie von den Tarifparteien weitgehend akzep- tiert und in der Durchführung als „unkompliziert" beschrieben (Bahnmüller/Hoppe 2011, S. 7). Dennoch gelten sie bei den Arbeitgebern nicht als zukunftsweisendes Instrument zur Lösung des Finanzierungsproblems in der Weiterbildung (vgl. auch im Folgenden Bahnmüller 2012). Diese ablehnende Haltung der Arbeitgeberseite führt dazu, dass auch die Gewerkschaftsseite wenig Anlass sieht, in dieser Richtung initiativ zu werden, zumal in der Mehrzahl der Qualifizierungstarifverträge die einzelbetriebliche Finanzierung der betrieblichen Weiterbildung durch den Arbeitgeber festgeschrieben ist und hiermit im Interesse der Beschäftigten ein Kompromiss erzielt wurde, von dem die Gewerkschaft ungern wieder abrückt. Ferner ist zu bedenken, dass tarifliche Fondslösungen zur Weiterbildungsfinanzierung bislang oft dann erst zustande kamen, wenn die Gewerkschaften ihrerseits auf andere Forderungen verzichteten. Angesichts der in den letzten Jahren teilweise eingetretenen Reallohnverluste gilt ein derartiger Entgeltverzicht zugunsten der Weiterbildung den Gewerkschaftsmitgliedern derzeit als kaum vermittelbar. Umlagefinanzierte Tariffonds zur Weiterbildungsfinanzierung werden derzeit denn auch nur von IG Metall und ver.di für die Leiharbeitsbranche diskutiert. Der Schwerpunkt gewerkschaftlicher Weiterbildungspolitik dürfte künftig eher in der Stär-

ÜBERSICHT 3

\section{Tarifliche Branchenfonds zur Weiterbildungsfinanzierung* in Deutschland}

\section{Textil- und Bekleidungsindustrie}

Im Wirtschaftsbereich Textil und Bekleidung haben sich die Tarifparteien von drei kleinen Branchen auf überbetriebliche Fondslösungen zur Weiterbildungsfinanzierung geeinigt.

\section{Miederindustrie}

Zu Beginn des Jahres 2012 trat die neu verhandelte Tarifvereinbarung zur überbetrieblichen Fondsfinanzierung der Weiterbildung in der Miederindustrie in Kraft. Erstmals wurde dieser Tariffonds im Jahr 1963 ausgehandelt und seitdem immer wieder verlängert. Hierbei verpflichten sich die Arbeitgeber, einen bestimmten Prozentsatz der Bruttolohn- und Gehaltssumme in einen Fonds zur Altersversorgung, Bildung und Gesundheitsförderung der Arbeitnehmer einzuzahlen.

\section{Textil Service}

In der BrancheTextil Service trat im Jahr 2009 ein Tarifvertrag in Kraft, bei dem der Industrieverband Textil Service-intex-e.V. und die IG Metall übereinkamen, "die Aus-, Fort-, Weiterbildung, präventiven Gesundheitsschutz sowie die Altersversorgung der Beschäftigten zu fördern." Die intex-Mitgliedsbetriebe verpflichten sich zu diesem Zweck, jährlich $35 €$ je Beschäftigten an einen Verein zur Verwaltung des Tariffonds abzuführen.

\section{Textil- und der Bekleidungsindustrie}

DerTarifvertrag zur Förderung von Aus-, Fort- und Weiterbildung der Beschäftigten trat im Jahr 1997 in Kraft. Zentrale Elemente dieses Tarifvertrags sind die Regelung eines individuellen Weiterbildungsanspruchs für die Beschäftigten bei gleichzeitiger Begrenzung des jährlichen Anspruchs auf maximal $2 \%$ der Belegschaft und die Einführung eines paritätisch verwalteten Bildungsfonds. Die Fondsmittel stehen jeweils zur Hälfte für arbeitgeberseitig und für arbeitnehmerseitig veranlasste Weiterbildung zur Verfügung (Bahnmüller/Jentgens 2005).

\section{Land- und Forstwirtschaft}

Im Jahr 2001 vereinbarten die Tarifparteien der Forstwirtschaft in Niedersachsen und der Land- und Forstwirtschaft in Schleswig-Holstein die Errichtung von Qualifizierungsfonds.

Die Modalitäten beiderTarifverträge basieren auf dem für das ostdeutscheTarifgebiet Mitte der 1990er Jahre vereinbarten tariflichen "Qualifizierungsfonds" der Land- und Forstwirtschaft.

Die Besonderheit dieses Fonds bestand darin, dass über ihn Qualifizierungsmaßnahmen gefördert wurden, die sich auch an ehemalige Beschäftigte in der Land- und Forstwirtschaft richteten und die eine Wiederaufnahme der land- und forstwirtschaftlichen Tätigkeit anstrebten. Mit Datum vom 10.11.2006 wurde der Qualifizierungsfonds der Land- und Forstwirtschaft e.V. liquidiert.

\section{Gerüstbaugewerbe}

Der finanziell bedeutendste Tariffonds ist die Sozialkasse im Gerüstbaugewerbe (Berger et al. 2012). Die Tarifvertragsparteien des Gerüstbaugewerbes vereinbarten dieses tarifliche Fondsmodell im Jahr 1981. Aus der Sozialkasse werden die tarifvertraglich geregelten Sozialleistungen einschließlich der Kosten für die berufliche Aus- und Weiterbildung finanziert. Förderfähig sind nach Tarifvertrag dieser Branche ausschließlich die Fortbildungslehrgänge zum geprüften GerüstbauKolonnenführer, zur Vorbereitung auf die Ausbildereignungsprüfung und zur $\mathrm{Ab}$ schlussprüfung Gerüstbauer/ Gerüstbauerin gemäß §45 Abs. 2 Berufsbildungsgesetz. 


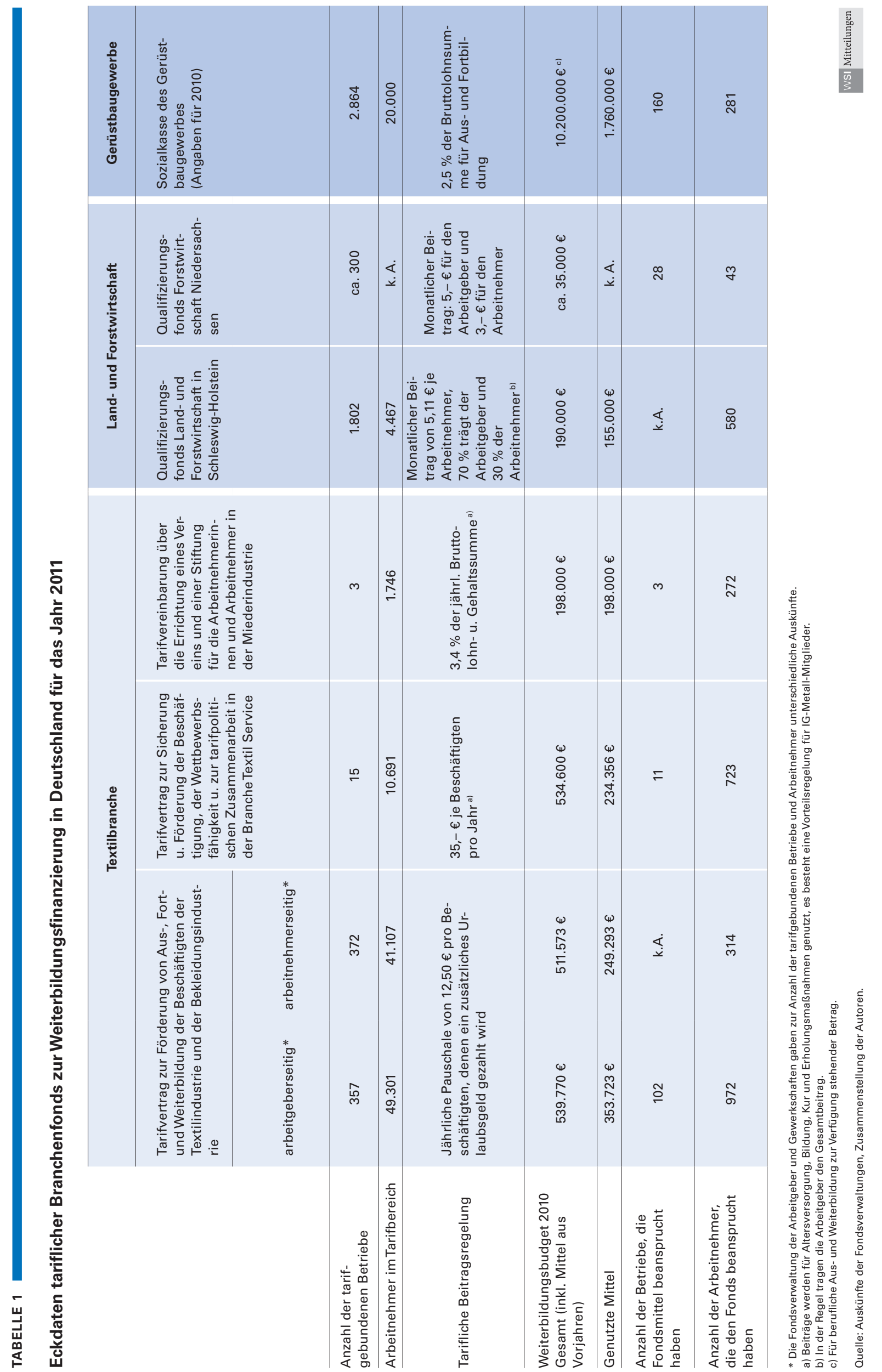


kung der Mitarbeiter- und Betriebsratsbeteiligung bei Bedarfsermittlung und Weiterbildungsplanung, bei der Stärkung der Weiterbildungsansprüche der Beschäftigten sowie in dem Ansatz liegen, Qualifizierungsfragen mit dem Entlohnungssystem zu verbinden.

\section{Tarifliche Weiterbildungspolitik in den Niederlanden}

Die tarifliche Weiterbildungspolitik ist in den Niederlanden in den letzten Jahrzehnten im Gegensatz zu Deutschland ein integrativer Teil des niederländischen Berufsbildungssystems geworden. Im Nachfolgenden wird die tarifliche Weiterbildungspolitik anhand ihrer zentralen Aspekte beschrieben.

\subsection{Genese}

Als Folge der rezessiven Phase in den Niederlanden Ende der 1970er Jahre stieg die (strukturelle) Arbeitslosigkeit stark an. Die Gewerkschaften und Arbeitgeberorganisationen vereinbarten in dieser Periode Maßnahmen, um Unternehmen und Beschäftigte zukünftig weniger anfällig für die ökonomische konjunkturelle Entwicklung zu machen (aktive Beschäftigungspolitik). Tarifverträge beinhalteten seit den 1960er Jahren sukzessive immer mehr Regelungen, die über die reinen tariflichen Regelungen von Löhnen hinausgehen. Bereits in den 1960er Jahren wurden solche tariflichen Freiräume (loonruimte) - unter anderen Voraussetzungen und aus anderen Gründen - in den Tarifverträgen vereinbart. Die Unternehmer zahlten 10 Gulden pro Beschäftigten in einen sektoralen Finanzierungstopf („loontientje“). Diese Gelder finanzierten damals u. a. bereits Qualifizierungsmaßnahmen. Anfang der 1980er Jahre wurden Tarifverträge vereinbart, die einen politischen Tausch zwischen Arbeitgeber und Gewerkschaften beinhalteten: Im „Akkoord van Wassenaar" (Hassel 1998) wurde eine restriktive Lohnentwicklung im Tausch für nicht lohnbasierte Zugeständnisse vereinbart. Mitte der 1980er Jahre entstanden daraus die sogenannten Sozialfonds. Als eine Form der Sozialfonds wurden die O\&O-Fonds (Ausbildungs- und Entwicklungsfonds) eingerichtet (Trampusch 2004). Diese Fonds sind zumeist tarifvertraglich geregelt und sollen teilweise zur Lösung eines zentralen Problems der Aus- und Weiterbildung beitragen (Poaching): Unternehmen, die nicht aus- und/oder weiterbilden, werben ausgebildete Fachkräfte von aus- und/ oder weiterbildenden Unternehmen ab (Waterreus 1997, 2002; Mooij/Houtkoop 2005; Donker van Heel et al. 2008).

\subsection{Reichweite}

1997 gab es 72 Branchenfonds (Waterreus 1997). 40 der 66 damals untersuchten Branchenfonds haben Maßnahmen der betrieblichen Weiterbildung unterstützt und durchge- führt. Diese Branchenfonds erfassten 1997 1,5 Mio. Beschäftigte und verfügten über Einnahmen in Höhe von 300 Mio. $€$, davon wurden 100 Mio. $€$ für Maßnahmen der betrieblichen Weiterbildung verwendet. Bis 2002 stieg die Zahl der Branchenfonds auf 99 und erreichte damit mehr als $40 \%$ aller Beschäftigten - ungefähr 2,5 Mio. (Waterreus 2002). Der jährliche Umsatz der Branchenfonds stieg auf 600 Mio. $€$ an, davon wurden zwei Drittel für Maßnahmen im Bereich der betrieblichen Weiterbildung, für die duale Lehrlingsausbildung und für Beschäftigungsprojekte genutzt. Die Maßnahmen im Bereich der betrieblichen Weiterbildung sind dabei mit 250 Mio. $€$ die wichtigste Ausgabenkategorie. Mitte 2007 gab es 140 Branchenfonds, von denen die übergroße Mehrheit an einen Tarifvertrag gebunden war. Die Anzahl der Fonds ändert sich ständig. Es werden neue gegründet, es kommt zu Fusionen und manchmal wird ein Fonds eingestellt. Von den 6,9 Mio. Beschäftigten in den Niederlanden fallen zurzeit insgesamt 5,9 Mio. oder $86 \%$ - in die Zuständigkeit eines O\&O-Fonds (Donker van Heel et al. 2008). Damit ist die tarifliche Weiterbildungspolitik ein zentraler Teil der beruflichen Weiterbildung geworden.

\subsection{Akteure}

Grundsätzlich gilt für die berufliche Bildung in den Niederlanden, dass die berufliche Erstausbildung in der kombinierten Verantwortung des Staates und der Tarifparteien liegt und die betriebliche Weiterbildung in der Verantwortung der Tarifparteien. Die Unternehmen bzw. die Tarifparteien sind also für die betriebliche Weiterbildung verantwortlich. In den Niederlanden gibt es im Gegensatz zu Frankreich keine nationale Gesetzgebung über die betriebliche Weiterbildung. Die Tarifverträge und auch die darin enthaltenen Sozialfonds (auch die Bildungsfonds) werden nachträglich vom Staat für die jeweiligen Branchen für allgemeinverbindlich erklärt, eine staatliche Einflussnahme auf die betriebliche Weiterbildung erfolgt damit eher indirekt.

\subsection{Akzeptanz}

Als Folge der wachsenden (strukturellen) Arbeitslosigkeit seit dem Ende der 1970er Jahre ergriff die niederländische Regierung in Zusammenarbeit mit den Tarifparteien eine Vielzahl von Maßnahmen. Neue Gesetze folgten in schnellem Tempo aufeinander. Die niederländische Arbeitsverwaltung wurde unter Beteiligung der Tarifparteien privatisiert und ein neues Gesetz zur Regelung der Erwachsenenbildung eingeführt. Auch die entstandenen O\&O-Fonds schafften Anreize für Arbeitgeber, in Bildung zu investieren. Durch die umlagefinanzierte Ausgleichsregelung beteiligen sich hier alle Unternehmen an der Finanzierung der Qualifizierung, auch diejenigen, die ihre Mitarbeiter nicht qualifizieren. Bildungsaktive Unternehmen erhalten dafür eine finanzielle Kompensation. Die in 30 Jahren gewachsene Akzeptanz der Branchenfonds ist groß. Dennoch wur- 
de vor einigen Jahren kritisiert, dass die Branchenfonds zu wenig von ihren Mitteln ausgeben. Um das Jahr 2000 hatten viele der Fonds große Reserven aufgebaut. Inzwischen sind diese Reserven aufgebraucht. Momentan hält die Mehrzahl der Fonds eine Mindestreserve in Höhe der erwarteten Ausgaben pro Jahr. Dies wurde durch einen proaktiven Ansatz erreicht, sowohl was die berufliche Qualifizierung und den Einsatz von Bildungsberatern betrifft als auch im Hinblick auf die Definition eines breiteren Aufgabenspektrums und durch Anpassungen bei der Höhe der Umlage, die die Unternehmen in Form eines Teils der Lohnsumme an die O\&O-Fonds abführen müssen.

\subsection{Kooperationsformen}

Die nationale Politik in den Niederlanden zielt zunehmend darauf ab, die Verantwortung im Bereich der beruflichen Bildung auf die regionalen und lokalen Behörden zu verlagern. Auf der regionalen Ebene sind die Berufsbildungszentren (ROC, regionale opleidingscentra), die lokalen $\mathrm{Ar}$ beitsverwaltungen und auch die Unternehmen die wichtigsten Akteure im Bereich der beruflichen Weiterbildung, die durch nationale (Wirtschaftsministerium, Branchenfonds) und/oder europäische Einrichtungen wie den Europäischen Sozialfonds (ESF) ko-finanziert werden. In einigen Fällen wurden verschiedene Formen regionaler Vereinbarungen entwickelt. Die meisten der bestehenden regionalen Vereinbarungen sind aber auf Branchenebene abgeschlossen (Branche-Region-Kombinationen). Die tarifliche Weiterbildungspolitik beinhaltet daher verschiedene Formen der Zusammenarbeit: Bildungsnetzwerke, gemeinsame Bildungspolitik, Bildungsvereinbarungen, Regelungen zur Steuerung von Bildungsangebot und -nachfrage, Finanzierungslösungen usw.

\subsection{Finanzierung}

Die bei Weitem wichtigste Finanzierungsquelle der O\&OFonds bilden die Abgaben der Unternehmen der jeweiligen Branche. 2005 lagen diese bei durchschnittlich 0,67 \% der Lohnsumme. Zwischen den Branchen und branchenintern gibt es große Unterschiede. So variiert in der Metallverarbeitungsbranche der für das Jahr 2010 geltende Prozentsatz zwischen 0,15\% für die Subbranche „Tankstellen und Autowaschanlagen“ und 1,0\% für die Subbranche der „Technischen Installationsbetriebe“. Die Prozentsätze werden im Rahmen von Tarifverhandlungen ausgehandelt und festgelegt. Eine zweite Finanzierungsquelle stellen die staatlichen Fördermittel dar. Dabei handelt es sich fast immer um eine zeitlich befristete Förderung. In den vergangenen Jahren waren dies beispielsweise Projektförderungen zur Verbesserung einer altersgerechten Personalpolitik sowie Mittel für das Zustandekommen von Vereinbarungen zum Gesundheits- und Arbeitsschutz. Eine dritte Finanzierungsquelle sind ESF-Mittel. Die Regierung hat beschlossen, dass diese Gelder, zumindest soweit es dabei um Projekte in den
Branchen und Unternehmen geht, über die O\&O-Fonds beantragt und verwaltet werden. Schätzungen zufolge investieren O\&O-Fonds pro Jahr etwa 600 Mio. €, davon 250 Mio. zur Förderung der Beschäftigungsfähigkeit bzw. Qualifizierung.

\subsection{Spektrum der Aktivitäten der O\&O-Fonds}

Die O\&O-Fonds können für unterschiedliche Aktivitäten in den Branchen genutzt werden, so z. B. für Information, Forschung, Beschäftigung von Arbeitslosen, Aus- und Weiterbildung. Zentrale Aufgabe ist allerdings die tätigkeitsbezogene Qualifizierung. Etwas mehr als 60 \% der Fonds setzen finanzielle Mittel ein, um Beschäftige in die Lage zu versetzen, die aktuelle Tätigkeit besser ausführen zu können. An zweiter Stelle, jedoch immer noch mit 50 \%, kommt die entwicklungsgerichtete Qualifizierung. Dabei handelt es sich um Qualifizierung, die im Prinzip den Übergang zu einer anderen, eventuell höherwertigen, Tätigkeit ermöglicht. Die Grenzen zwischen diesen beiden Formen der Qualifizierung sind jedoch fließend. An dritter Stelle stehen mit 37 \% Verfahren zur Feststellung non-formal und informell erworbener beruflicher Kompetenzen. Sie ermöglichen den Beschäftigten, die während ihrer beruflichen Tätigkeit erworbenen Kenntnisse und Fertigkeiten formal anerkennen zu lassen, was den Übergang in eine andere Tätigkeit in der eigenen Branche oder den Wechsel in eine andere Branche vereinfacht. Die sektoralen O\&O-Fonds unterstützen auch vermehrt Bildungsmaßnahmen für Beschäftigte, die in eine andere Branche wechseln. In einigen Branchen wurden zusätzliche Vereinbarungen verabschiedet. Diese umfassen u. a. Fonds für spezifische Zielgruppen, wie z. B. Frauen, Langzeitarbeitslose, Beschäftigte ohne oder mit einer geringen Ausbildung oder ausländische Beschäftigte. Die Tarifparteien in den Branchen, in denen Bildungsvereinbarungen getroffen wurden, sind in die Aus- und Weiterbildung stark involviert. Die sektoralen O\&O-Fonds sind außerdem ein wichtiges Instrument zur Förderung der betrieblichen Weiterbildung in Klein- und Mittelunternehmen. In den Anfangsjahren der Bildungsfonds war die Nutzung durch Klein- und Mittelunternehmen noch gering. Dies hat sich seit Ende der 1990er Jahre deutlich verbessert. Insgesamt sind das Weiterbildungsangebot und die Weiterbildungsteilnahme in den Niederlanden und besonders auch in den Klein- und Mittelunternehmen stark gestiegen.

\section{Ist die fondsfinanzierte tarifliche Weiterbildungspolitik für Deutsch- land eine politische Option?}

Im Vergleich mit den europäischen Ländern, die eine ähnliche sozio-ökonomische Struktur aufweisen, ist die betriebliche Weiterbildung in Deutschland deutlich schlechter 
aufgestellt als in den Niederlanden. In diesem Beitrag wurde argumentiert, dass dies daran liegen kann, dass das jeweilige System der beruflichen Weiterbildung eher kooperativ ausgerichtet oder eher segmentiert ist.

Zwischen den beiden Ländern existieren deutliche Unterschiede bei der Kooperation und Koordination der Tarifparteien und des Staates in der beruflichen Bildung. Während in Deutschland eine ausgeprägte, gesetzlich festgelegte Zusammenarbeit der Gewerkschaften und Arbeitgeberverbände in der beruflichen Ausbildung besteht, kooperieren diese Akteure bei der beruflichen Weiterbildung nur im Bereich der wenigen staatlich anerkannten Fortbildungsabschlüsse miteinander (segmentiertes System der beruflichen Weiterbildung). In den Niederlanden arbeiten Staat und Tarifparteien auf der Grundlage allgemeingültig erklärter tariflicher Vereinbarungen sowohl in der beruflichen Ausbildung als auch in der Weiterbildung zusammen (kooperatives System der beruflichen Weiterbildung).

Die tarifliche Weiterbildungspolitik und ihre Fondsfinanzierung in den Niederlanden bilden die Grundlage für eine koordinierte Verantwortlichkeit und Zusammenarbeit aller an der beruflichen Bildung beteiligten Akteure. Ihre Entstehung war letztlich nur denkbar vor dem Hintergrund einer erheblichen strukturellen (Jugend-)Arbeitslosigkeit Anfang der 1980er Jahre. Erst unter diesem Druck einigten sich Tarifparteien und Staat darauf, sozialpolitischen Maßnahmen und insbesondere auch Qualifizierungsmaßnahmen den Vorrang vor Lohnerhöhungen zu geben. Auf Grundlage dieses Sozialpaktes entwickelte sich in den letzten drei Jahrzehnten unter Beteiligung des Staates eine tarifliche Weiterbildungspolitik, die bis heute die Durchführungsbedingungen für das niederländische Berufsbildungssystem bestimmt.

Hingegen führte der ökonomisch-technologische Strukturwandel in Deutschland nur in wenigen kleinen Branchen zu umlagefinanzierten Tariffonds. Ähnlich wie in den Niederlanden wurden aber auch diese meist durch Verzicht, z. B. auf eine Erhöhung des Urlaubsgeldes, möglich. Dort, wo sie existieren, haben sie teilweise eine lange Tradition und sind von den Tarifparteien weitgehend akzeptiert. In der Durchführung gelten sie als unkompliziert und tragen gerade auch in klein- und mittelbetrieblich geprägten Branchen dazu bei, die Qualifizierung von Fachkräften nicht mehr als einzelbetriebliches Problem, sondern als Herausforderung für die gesamten Branche zu erkennen.

Anders als in den Niederlanden, in denen der Staat an der nationalen Tarifpolitik aktiv beteiligt ist, werden Tarifvereinbarungen in Deutschland ausschließlich von den Tarifparteien in den jeweiligen Tarifbereichen geschlossen. Ein Transfer des niederländischen Modells ist schon wegen der im Grundgesetz abgesicherten Tarifautonomie, aber auch wegen der sektoralen Struktur der tariflichen Weiterbildungspolitik in Deutschland nicht möglich. Eine Bedingung für eine weitere Ausbreitung sektoraler Tariffonds ist also, dass die Tarifparteien selbst hierin eine zukunftswei- sende Handlungsoption erblicken (Trampusch/Eichenberger 2011). Bahnmüller (2012) weist in einer aktuellen Expertise darauf hin, dass Fondsmodelle in den großen Tarifbereichen der Metall- und Elektroindustrie von Arbeitgeberseite nach wie vor abgelehnt werden. Auch für große Gewerkschaften wie die IG Metall in Baden-Württemberg stellen sie derzeit keine tarifpolitische Option dar.

Der direkte politische Transfer einer tariflichen Weiterbildungspolitik, wie sie in den Niederlanden in den letzten Jahrzehnten gewachsen ist, scheint daher für die deutschen Akteure im Bereich der beruflichen Weiterbildung keine realistische Option zu sein. Dennoch wird ein möglicher Beitrag der Tarifpolitik zur Weiterbildungsfinanzierung in Deutschland immer wieder diskutiert. So wurde vom Bundesministerium für Bildung und Forschung (BMBF) bereits im Jahr 2001 eine Expertenkommission „Finanzierung lebenslangen Lernens" eingesetzt, die neue Förder- und Finanzierungskonzepte entwickeln sollte (BMBF 2004). Die 2004 veröffentlichten Empfehlungen der Kommission gehen dabei nicht nur von einem Handlungsbedarf des Staates und der Individuen aus, auch sei „der Betrieb [...] für die meisten erwerbstätigen Erwachsenen der wichtigste Lernort “ (BMBF 2004, S. 236). Die Kommission forderte deshalb „die Tarifparteien und die betrieblichen Partner auf, die Instrumente der Arbeits- und Lernzeitkonten intensiv zu nutzen und ggf. auf tarifvertraglicher Ebene kollektive Finanzierungsregeln zu entwickeln, wie sie etwa in der Baubranche bekannt sind“ (BMBF 2004, S. 290). Während die Empfehlung an die Tarifparteien, tarifliche Regelungen zu Lernzeiten zu vereinbaren, im Konsens erfolgte, blieb das Instrument tariflicher Finanzierungsregelungen in der Kommission umstritten. Lediglich für die Leiharbeitnehmer im expandierenden Zeitarbeitsmarkt empfahl die Kommissionsmehrheit die Einrichtung tariflicher Branchenfonds. Umlagefinanzierte Tariffonds zur Weiterbildungsfinanzierung werden derzeit nur von IG Metall und ver.di für die Leiharbeitsbranche diskutiert (Bahnmüller 2012). Jüngst hat Bosch (2012) angesichts des drohenden Fachkräftemangels und sinkender Weiterbildungsinvestitionen in Deutschland erneut vorgeschlagen, über eine Fondsfinanzierung nachzudenken. Dazu hat er ein Modell eines Weiterbildungsfonds für Deutschland entwickelt, in dem sowohl die betriebliche als auch die individuelle Weiterbildung durch Umlage- und Zuschussfonds gefördert werden sollen.

Auch von Seiten der Europäischen Union gibt es aktuell Initiativen, die tarifliche Weiterbildungspolitik zu fördern. Mit Inkrafttreten der Sozialpartnerrichtlinie am 26. März 2009 wurde in Deutschland erstmalig im Rahmen der ESFFörderperiode von 2007-2013 eine finanzielle Förderung tariflicher Vereinbarungen ermöglicht, die die Tarifparteien zur Verbesserung der beruflichen Weiterbildung und deren Rahmenbedingungen abgeschlossen haben (BMAS 2009). Qualifizierungstarifverträge werden damit als wichtiges Instrument zur Förderung der betrieblichen Weiterbildung hervorgehoben. Der Schwerpunkt der hierüber geförderten Qualifizierungsvereinbarungen liegt bei 
der Regelung der Bedarfsermittlung und Planung der betrieblichen Weiterbildung. Hinsichtlich der Finanzierung haben sich die Tarifparteien meist auf folgende Lastenteilung geeinigt: Betriebliche Weiterbildung zahlt der Arbeitgeber. Für „persönliche berufliche“ Weiterbildung kommen die Beschäftigten auf.

\section{LITERATUR}

Bahnmüller, R. (2009): Tarifverträge als Instrument der beruflichen (Weiter-)Bildung in Deutschland. Shanghai, http://www.regiestelle-weiterbildung.de/fileadmin/pdfs/anfahrt/Bahnmueller.pdf (letzter Zugriff: 16.05.2012)

Bahnmüller, R. (2012): Tarifliche Weiterbildungsregelungen für die M+E-Industrie: Regulierungsansätze, Wirkungen und Perspektiven einer überbetrieblichen Weiterbildungsfinanzierung, unveröffentlichtes Projektmanuskript Bahnmüller, R./Fischbach, S. (2006): Qualifizierung und Tarifvertrag. Befunde aus der Metallindustrie Baden-Württembergs, Hamburg 2006

Bahnmüller, R./Hoppe, M. (2011): Überbetriebliche und betriebsbezogene tarifvertragliche Qualifizierungsregelungen in Deutschland: Ein Überblick in 13 Punkten, BIBB-Konferenz 2011, Sektion 5.2., Überbetriebliche Zusammenarbeit in der Weiterbildungsfinanzierung im In- und Ausland

Bahnmüller, R./Jentgens, B. (2005): Expertise zur Nutzung und zu den Wirkungen des Tarifvertrags zur Aus-, Fort- und Weiterbildung in der westdeutschen Textil- und Bekleidungsindustrie, Tübingen

Behringer, F./Moraal, D./Schönfeld, G.(2008): Betriebliche Weiterbildung in Europa: Deutschland weiterhin nur im Mittelfeld. Aktuelle Ergebnisse aus CVTS3, in: Berufsbildung in Wissenschaft und Praxis 37 (1), S. 9-14

Berger, K./Häusele, S./Moraal, D. (2012): Tarifvertraglich geregelte Finanzierung der beruflichen Weiterbildung am Beispiel der Sozialkasse im Gerüstbaugewerbe, in: Berufbildung in Wissenschaft und Praxis 41 (2), S. 49-53 Bosch, G. (2012): Weiterbildungsfonds - ein Finanzierungsmodell auch für Deutschland, in: Berufsbildung in Wissenschaft und Praxis 10 (1), S. 23-26 Bundesministerium für Arbeit und Soziales (BMAS) (Hrsg.) (2009): ESF-Richtlinie zur Förderung der beruflichen Weiterbildung von Beschäftigten (Sozialpartnerrichtlinie), in: Bundesanzeiger Ausgabe Nr. 57 vom 16. April, S. 1373 Bundesministerium für Bildung und Forschung (BMBF) (Hrsg.) (2004): Schlussbericht der Expertenkommission Finanzierung Lebenslangen Lernens:Der Weg in die Zukunft. 28. Juli, http://www.bmbf.de/pub/ schlussbericht_kommission_III.pdf (letzter Zugriff 03.02.2009)

Busemeyer, M. R. (2009): Wandel trotz Reformstau. Die Politik der beruflichen Bildung seit 1970, Schriften aus dem MPI für Gesellschaftsforschung, Bd. 65, Frankfurt a. M.

Busemeyer M. R./Trampusch, C. (Hrsg) (2011): The Political Economy of Collective Skill Formation, Oxford
Donker van Heel, P./van Velden, J./Siegert, J./Groenendijk, R./de Kogel, M./ Sincer, E. (2008): Hoe werken sectorfondsen?, Rotterdam, http://www. flexservice.com/wp-content/uploads/Hoe_werken_sectorfondsen.pdf (letzter Zugriff: 04.11.2011)

Hassel, A. (1998): Soziale Pakte in Europa, in: Gewerkschaftliche Monatshefte 49 (10), S. $626-637$

Mooij, A. de/Houtkoop, W. (2005): Scholing op afspraak. Sectorale scholingsafspraken en stand van zaken O\&O fondsen, Amsterdam

Moraal, D. (2007): Berufliche Weiterbildung in Deutschland, Bundesinstitut für Berufsbildung, Bonn, http://www.bibb.de/de/30130.htm (letzter Zugriff: 16.05.2012)

Sauter, E. (1989): Ansätze für eine Neuorientierung der beruflichen Weiterbildung, in: Berufsbildung in Wissenschaft und Praxis 18 (3), S. 3-8

Trampusch, C. (2004): Sozialpolitik durch Tarifvertrag in den Niederlanden: Die Rolle der industriellen Beziehungen in der Liberalisierung des Wohlfahrtsstaates, MPIfG Discussion Paper (12), Köln

Trampusch, C./Busemeyer,M.R. (2011):The Political Economy of Collective Skill Formation, Oxford

Trampusch, C./Eichenberger, P. (2011): Skills and Industrial Relations in Coordi nated Market Economies - Continuing Vocational Training in Denmark, the Netherlands, Austria, and Switzerland, in: British Journal of Industrial Relations, Oxford (im Erscheinen)

Waterreus J. M. (1997): O\&O fondsen onderzocht. Opleidings, en ontwikkelingsfondsen en de scholing van werknemers, Amsterdam

Waterreus J. M. (2002): O\&O fondsen op herhaling. Stand van zaken scho lingsfondsen 2002, Amsterdam

\section{AUTOREN}

KLAUS BERGER, Dipl. Päd, ist wissenschaftlicher Mitarbeiter im Bundesinstitut für Berufsbildung (BIBB). Arbeitsschwerpunkte: Kosten, Nutzen, Finanzierung der Berufsbildung.

berger@bibb.de

DICK MORAAL, Dipl. Pol., ist wissenschaftlicher Mitarbeiter im BIBB. Arbeitsschwerpunkte: Kosten, Nutzen, Finanzierung. Arbeitsschwerpunkte: Betriebliche Weiterbildung im internationalen Vergleich, Kosten der betrieblichen Ausund Weiterbildung.

moraal@bibb.de 